\title{
RPN11 deubiquitinase promotes proliferation and migration of breast cancer cells
}

\author{
GUOQING LUO ${ }^{1,2}$, NINGDONG HU ${ }^{2}$, XU XIA $^{2}$, JINGJING ZHOU ${ }^{2}$ and CHANGSHENG YE ${ }^{1}$ \\ ${ }^{1}$ Breast Center, Nanfang Hospital, Southern Medical University, Guangzhou, Guangdong 510515; \\ ${ }^{2}$ The First Zone of Cardiothoracic Department, Qingyuan People's Hospital, The Sixth Affiliated \\ Hospital of Guangzhou Medical University, Qingyuan, Guangdong 511518, P.R. China
}

Received March 19, 2016; Accepted February 21, 2017

DOI: $10.3892 / \mathrm{mmr} .2017 .6587$

\begin{abstract}
The deubiquitinase enzyme RPN11 is involved in oncogenesis in various types of cancer. However, in breast cancer, the expression levels, prognostic relevance and biological function of RPN11 remains unclear. In the present study, RPN11 expression levels in breast cancer tissues and adjacent non-tumor tissues were determined by reverse transcription-quantitative polymerase chain reaction and immunohistochemical staining, and the association of RPN11 with clinicopathological features of breast cancer was evaluated. RPN11 expression was upregulated in breast cancer tissues compared with healthy tissues. Additionally, high expression levels of RPN11 may be an indicator of poor prognosis, as validated by a breast cancer cohort from the Gene Expression Omnibus database. Knockdown of RPN11 in MDA-MB-231 and T47D cells significantly reduced cell proliferation and enhanced G0/G1 arrest and apoptosis. Exogenous overexpression of RPN11 in MCF7 and Hs578T cells promoted cell growth and inhibited apoptosis. In addition, knockdown of RPN11 abrogated cell migration and reduced epithelial-mesenchymal transition. In conclusion, these findings suggested that RPN11 may function as an oncogene and its upregulation in breast cancer suggests that it may be a therapeutic target.
\end{abstract}

\section{Introduction}

Breast cancer (BC) is the most frequently diagnosed malignancy and is one of the leading causes of mortality in women, with nearly a quarter of million new cases occurring in the United States in 2016 (1,2). The heterogeneity of breast cancer is a primary factor that contributes to the intractable clinical treatments. For example, hormone therapy is available for luminal A or B breast cancers that are estrogen (ER)/progesterone (PR) positive. However, there are limited available

Correspondence to: Dr Changsheng Ye, Breast Center, Nanfang Hospital, Southern Medical University, 1838 North Guangzhou Avenue, Guangzhou, Guangdong 510515, P.R. China

E-mail: georgeye11@sina.cn

Key words: RPN11, breast cancer, proliferation, migration treatments for breast cancer that is triple-negative for ER, PR and human epidermal growth factor receptor 2 (HER2). Numerous molecular biomarkers and signaling pathways have been investigated, including aberrant activation of Akt and Wnt signaling pathways, in addition to changes in microRNAs and oncogenes (3-6). However, the mechanisms underlying the development of breast cancer require further understanding, particularly the development of malignant phenotypes.

The deubiquitinating enzyme RPN11, alternatively known as proteasome $26 \mathrm{~S}$ subunit non-ATPase 14 or POH1, is a component of the $26 \mathrm{~S}$ proteasome and reverses the effects of the ubiquitin-proteasome degradation system. RPN11 functions in diverse biological processes, including DNA repair, embryonic cell development and differentiation, programmed cell death and drug resistance (7-9). RPN11 has been reported to modulate the proliferation of tumor cells by regulating the phosphorylation of retinoblastoma protein and cyclin-dependent kinases (10). In addition, RPN11 was significantly upregulated in hepatocellular carcinoma and promoted tumorigenesis by stabilizing E2F transcription factor 1 (E2F1) and its target genes (11). Gallery et al (12) reported that the JAB1/MPN/Mov34 metalloenzyme motif of RPN11 was essential for cell viability. A previous study has suggested that RPN11 may regulate the ErbB2 receptor and c-jun ubiquitination, and enhance the transcriptional activity of microphthalmia-associated transcription factor (13-15). However, there is currently no evidence for the potential role of RPN11 in breast cancer development and treatment.

The present study reports that mRNA and protein expression levels of RPN11 were overexpressed in breast cancer tissue. RPN11 significantly promoted tumor cell proliferation and inhibited apoptosis. In addition, knockdown of RPN11 inhibited cell migration by suppressing epithelial-mesenchymal transition (EMT). Therefore, the results of the present study demonstrate that inhibition of RPN11 may serve as a treatment for breast cancer.

\section{Materials and methods}

Tissue specimens. A total of 59 breast cancer tissues and paired noncancerous tissues were obtained from the First Zone of Cardiothoracic Department, Qingyuan People's Hospital (Qingyuan, China). The study was approved by the 
Ethical Committee of Qingyuan People's Hospital and the written informed consent was obtained. The patients did not receive radiotherapy or chemotherapy prior to tissue collection. In addition, six pairs of fresh breast cancer tissues and adjacent noncancerous tissues were utilized for western blot analysis. Tissues were stored at $-80^{\circ} \mathrm{C}$ prior to processing. A tissue microarray containing 142 cases of breast cancer specimens with complete clinicopathological features was utilized in this study and was obtained from Shanghai Outdo Biotech Co. Ltd (cat. no. HBre-Duc150Sur-02; Shanghai, China). The remaining patients with breast cancer were obtained from GEO database (www. ncbi.nlm.nih.gov/).

Cell lines. MCF7, MDA-MB-231, Hs578T and T47D human cancer cell lines were obtained from the American Type Culture Collection (Manassas, VA, USA) and maintained in Dulbecco's modified Eagle's medium supplemented with 10\% fetal bovine serum, $100 \mu \mathrm{g} / \mathrm{ml}$ streptomycin and $100 \mathrm{U} / \mathrm{ml}$ penicillin at $37^{\circ} \mathrm{C}$ and $5 \% \mathrm{CO}_{2}$.

PlasmidandsiRNAtransfection.The pcDNA3.1-RPN11 plasmid was utilized for overexpression of RPN11 and was obtained from Guangzhou RiboBio Co., Ltd. (Guangzhou, China). Cells at a density of $10^{6}$ cells/well were plated into the 6-well plate and transfected with pcDNA3.1-RPN11 or vector control using Lipofectamine $^{\circledR} 2000$ (Invitrogen; Thermo Fisher Scientific, Inc., Waltham, MA, USA). For siRNA transfection, cells were transfected with a pool of three RPNII siRNAs or a negative control siRNA. siRNAs targeting RPN11 were obtained from Chang Jing Bio-Tech, Ltd. (Changsha, China). The sequences were as follows: Negative control siRNA, 5'-UUCUCCGAA CGUGUCACGUTT-3'; RPNI1 siRNA-1, 5'-CAAGTTAAA TCTAGCTCAA-3'; RPN11 siRNA-2, 5'-GCAAGACAAGGG TCCATAT-3' and RPN11 siRNA-3, 5'-TAAGACATCTGGCAT CATT-3'. The cells were cultured for $48 \mathrm{~h}$.

Reverse transcription-quantitative polymerase chain reaction $(R T$ - $q P C R)$. Total RNA was isolated using TRIzol ${ }^{\circledR}$ (Invitrogen; Thermo Fisher Scientific, Inc.) according to the manufacturer's protocol. cDNA was synthesized using the PrimeScript RT Master kit (Takara Biotechnology Co., Ltd., Dalian, China). RT-qPCR was performed on a 7500 Real-Time PCR system (Applied Biosystems, Thermo Fisher Scientific, Inc.) using SYBR $^{\circledR}$-Green. Primers were as follows: Forward, $5^{\prime}$-TGCTAT GCCACAGTCAGGAA-3' and reverse, 5'-ACAACCATCTCC GGCCTTC-3' for human RPN11; forward, 5'-GTCTCCTCT TGGCTCTGCC-3' and reverse, 5'-AAATTCACTCTGCCC AGGACG-3' for human E-cadherin; forward, 5'-GAGGCT TCTGGTGAAATCGC-3' and reverse, 5'-TGCAGTTGCTAA ACTTCACATT-3' for human N-cadherin; forward, 5'-AAT CCAGAGTTTACCTTCCAGCA-3' and reverse, 5'-TCCCAG ATGAGCATTGGCAG-3' for human Snail; forward, 5'-GAA CTGGACACACATACAGTGATT-3' and reverse, 5'-AGT GATGGGGCTGTATGCTC-3' for human Slug; forward, 5'-CGGGAGAAATTGCAGGAGGA-3' and reverse, 5'-AAG GTCAAGACGTGCCAGAG-3' for human vimentin; forward, 5'-ACAAACACTAATGTTAATTGCCCA-3' and reverse, 5'-TCTTGGCAGAGAGACATGCTT-3' for human fibronectin 1. Human GAPDH served as an internal control: GAPDH forward, 5'-CATGAGAAGTATGACAACAGCCT-3' and reverse, 5'-AGTCCTTCCACGATACCAAAGT-3'. The PCR conditions were as follows: $95^{\circ} \mathrm{C} 30 \mathrm{sec}, 95^{\circ} \mathrm{C} 30 \mathrm{sec}, 60^{\circ} \mathrm{C}$ $30 \mathrm{sec}$ for 40 cycles. The expression levels of indicated genes were normalized using the $2^{-\Delta \Delta C q}$ method relative to the human GAPDH gene (16).

Western blot analysis. Tissue samples and cells were lysed in radioimmunoprecipitation assay buffer (Thermo Fisher Scientific, Inc.) containing protease and phosphatase inhibitor cocktail (Roche Diagnostics, Basel, Switzerland). Protein concentrations were determined by the bicinchoninic acid assay. A total of $60 \mu \mathrm{g}$ lysate was loaded onto $12 \%$ gels, subjected to SDS-PAGE and transferred onto polyvinylidene fluoride membranes. Membranes were blocked with 5\% BSA (Sangon Biotech Co., Ltd., Shanghai, China) were incubated with primary antibody overnight at $4^{\circ} \mathrm{C}$, washed and probed with HRP-labeled goat-anti-rabbit secondary antibody (dilution, 1:1,000; cat. no. ab6721; Abcam, Cambridge, MA, USA) at room temperature for $2 \mathrm{~h}$. Proteins were visualized using ECL chemiluminescent detection substrate (Thermo Fisher Scientific, Inc.), according to the manufacturer's protocol.Primary antibodies, diluted at 1:1,000 were as follows: rabbit anti-RPN11 (catalog no. 12059-1-AP; ProteinTech Group, Inc., Chicago, IL, USA), mouse anti-tubulin (catalog no. sc-5286; Santa Cruz Biotechnology, Inc., Dallas, TX, USA), rabbit anti-cleaved PARP (catalog no. 5625) and mouse anti-snail (catalog no. 3895; Cell Signaling Technology, Inc., Danvers, MA, USA), rabbit anti-E-cadherin (catalog no. 1702), rabbit anti-N-cadherin (catalog no. 2447), rabbit anti-cyclin D1 (catalog no. 2261) and rabbit anti-vimentin (catalog no. 2707; Epitomics, Burlingame, CA, USA).

Cell viability assay. The rate of cell proliferation was measured by MTT assay. The cells were transfected with RPN11 cDNA and vector as control, or siRNA and NC as control. Subsequently, the cells were seeded in 96 -well plates to a density of 1,000 cells/well. MTT reagent $(5 \mathrm{mg} / \mathrm{ml})$ was added to the medium at various time points. Following $3 \mathrm{~h}$ incubation, the medium was discarded and DMSO was added to form a precipitate. The absorbance was measured at a wavelength of $540 \mathrm{~nm}$.

Flow cytometric assay. Cells were seeded at $10^{5}$ fixed with $70 \%$ ethanol and stained with propidium iodide (Sigma-Aldrich; Merck KGaA, Darmstadt, Germany) and RNase (Takara Biotechnology Co., Ltd.) for $30 \mathrm{~min}$. Subsequently, the distribution of the cell cycle was measured by flow cytometry and analyzed using ModFit LT ${ }^{\mathrm{TM}}$ software version 3.2 (Verity Software House, Topsham, ME, USA). In addition, cell apoptosis was analyzed by flow cytometry following Annexin V/7-aminoactinomycin D (AAD) staining kit (Thermo Fisher Scientific, Inc.). Cells at a density of $10^{5}$ were cultured in 6-well plates, harvested and resuspended in binding buffer. Following staining with Annexin V and 7-AAD for 20 min, cells were analyzed using a flow cytometer.

Transwell assay. Cells were starved in serum free medium for $24 \mathrm{~h}$ and seeded on the top chamber of a Transwell plate at $1 \times 10^{4}$ cells/well. The bottom chambers were filled with medium supplemented with $20 \%$ FBS. After 48 h, cells remaining in the top chamber were carefully removed and the lower surface was fixed in $4 \%$ paraformaldehyde and stained 
A

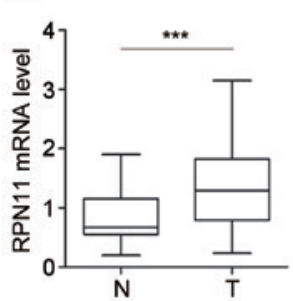

B GSE3744

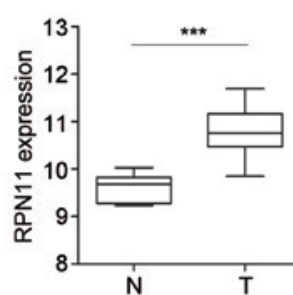

C

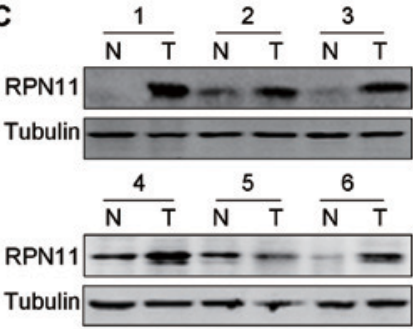

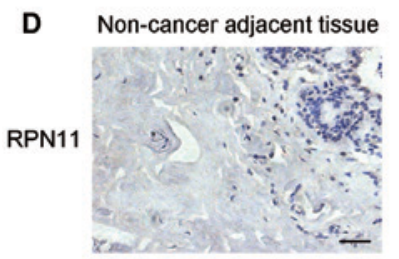

Tumor 1

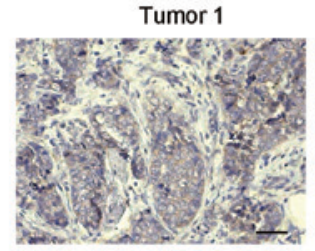

Tumor 2

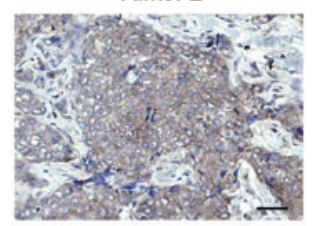

E
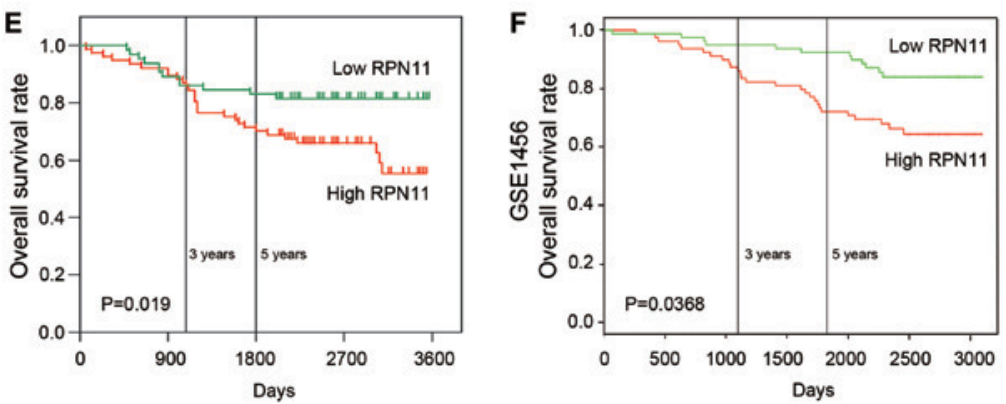

Figure 1. RPN11 is overexpressed in breast cancer tissues and associated with poor prognosis. (A) Relative mRNA levels of RPN11 in 59 cases of paired breast cancer tissue and adjacent non-cancer tissues were measured by reverse transcription-quantitative polymerase chain reaction. (B) Expression levels of RPN11 were measured according to the GSE3744 dataset in the Gene Expression Omnibus database. Data are expressed as the mean \pm standard error of three independent experiments. ${ }^{* * *} \mathrm{P}<0.001$. (C) Protein expression levels of RPN11 was measured by western blot analysis in 6 cases of breast cancer and adjacent non-cancer tissues. (D) Representative images of RPN11 following immunohistochemical staining in breast cancer and healthy tissue. Scale bar: $100 \mu \mathrm{m}$. (E) The prognostic value of low and high RPN11 expression was measured by Kaplan-Meier survival curves in 142 cases of breast cancer and (F) the GSE1456 dataset. N, non-cancer adjacent tissue; T, tumor.

with $1 \%$ crystal violet. Cells were counted and imaged in five random fields using a phase contrast microscope. Experiments were repeated at least three times.

Immunohistochemical staining (IHC). Tissue microarray slides were deparaffinized, rehydrated in ethanol and subjected to antigen retrieval by heating in $0.01 \mathrm{M}$ citrate buffer (Beyotime Institute of Biotechnology, Haimen, China), to block endogenous peroxidases. Following washing in PBS and blocking with $5 \%$ bovine serum albumin (Sangon Biotech Co., Ltd.), the slides were incubated with a primary RPN11 antibody (cat. no. 12059-1-AP; ProteinTech Group, Inc.; 1:500 dilution) overnight at $4^{\circ} \mathrm{C}$. Following three washes in PBS, slides were incubated with a horseradish peroxidase-conjugated secondary antibody (cat. no. AR1022; 1:500; Boster Biological Technology, Ltd., Wuhan, China) for $1 \mathrm{~h}$ at room temperature and subsequently washed three times in PBS. Tissues were stained with diaminobenzidine and counterstained with hematoxylin and observed using a light microscope.

Tissues were evaluated by two pathologists and scored according to the percentage and intensity of staining, as previously described (17). Briefly, the percentage score was as followed: i) $0-5 \%$; ii) $6-50 \%$; iii) $51-75 \%$ and iv) $76-100 \%$. The intensity score was as followed: i) No staining; ii) weak; iii) moderate and iv) strong. A final score from 1 to 16 was calculated by multiplying the percentage score by the intensity score. For each sample, $\leq 8=$ low expression and $>8=$ high expression.

Statistical analysis. Statistical calculations were performed using SPSS version 21.0 software (IBM SPSS, Armonk, NY, USA). PROGgene version 2.0 software (Indiana University, Indianapolis, IN, USA) was used to evaluate the prognosis of indicated types of cancer. Clinical parameters were compared with RPN11 expression in tissue samples by the $\chi^{2}$ test. Kaplan-Meier curves were utilized to determine the overall survival distribution. Student's t-test was performed to compare the significance between the control group and experimental groups. Data are expressed as the mean \pm standard error of three independent experiments. $\mathrm{P}<0.05$ was considered to indicate a statistically significant difference.

\section{Results}

Elevated RPN11 expression in breast cancer is associated with poor prognosis. To determine the potential role of RPN11 in breast cancer pathogenesis, the mRNA expression levels of RPN11 was determined in 59 cases of paired tumor and adjacent non-tumorous tissues. Greater expression levels of RPN11 were detected in breast cancer tissues compared with non-cancer adjacent tissue $(\mathrm{P}<0.001$; Fig. 1A). Subsequently, 
Table I. RPN11 expression status and clinicopathological features of breast cancer tissue samples.

\begin{tabular}{|c|c|c|c|c|c|}
\hline \multirow[b]{2}{*}{ Clinicopathological feature } & \multirow[b]{2}{*}{ No. of cases } & \multicolumn{2}{|c|}{ RPN11 expression } & \multirow[b]{2}{*}{$\chi^{2}$} & \multirow[b]{2}{*}{ P-value } \\
\hline & & Negative & Positive & & \\
\hline Age & & & & 0.333 & 0.564 \\
\hline$\leq 55$ & 64 & 31 & 33 & & \\
\hline$>55$ & 78 & 34 & 44 & & \\
\hline Tumor size $(\mathrm{cm})$ & & & & 2.849 & 0.241 \\
\hline$<2$ & 19 & 12 & 7 & & \\
\hline$\geq 2$ and $<5$ & 100 & 44 & 56 & & \\
\hline$\geq 5$ & 23 & 9 & 14 & & \\
\hline Lymphatic vessel invasion & & & & 2.886 & 0.089 \\
\hline Yes & 70 & 27 & 43 & & \\
\hline No & 72 & 38 & 34 & & \\
\hline $\mathrm{T}$ classification & & & & 3.071 & 0.080 \\
\hline $\mathrm{T}_{0-1}$ & 38 & 22 & 16 & & \\
\hline $\mathrm{T}_{2-4}$ & 104 & 43 & 61 & & \\
\hline $\mathrm{N}$ classification & & & & 3.511 & 0.061 \\
\hline $\mathbf{N}_{0-1}$ & 110 & 55 & 55 & & \\
\hline $\mathrm{N}_{2-3}$ & 32 & 10 & 22 & & \\
\hline Tumor stage & & & & 6.435 & $0.040^{\mathrm{a}}$ \\
\hline I & 24 & 14 & 10 & & \\
\hline II & 80 & 40 & 40 & & \\
\hline III+IV & 38 & 11 & 27 & & \\
\hline
\end{tabular}

${ }^{\mathrm{a}} \mathrm{P}<0.05$.

the GSE3744 dataset was analyzed, which contained 47 human breast tumor cases and 7 cases of non-cancerous tissues. The expression levels of RPN11 were greater in tumor tissues compared with healthy tissues $(\mathrm{P}<0.001$; Fig. 1B). Western blot analysis demonstrated elevated RPN11 expression levels in six breast cancer tissues compared with adjacent non-cancerous tissues (Fig. 1C).

IHC was performed on tissue microarrays to measure RPN11 expression in 142 tissues derived from breast cancer patients, whose 5-year follow-up data were available. Low levels of RPN11 were detected in adjacent non-cancerous tissues, whereas high levels of RPN11 staining were observed in breast cancer tissues (Fig. 1D). In addition, RPN11 expression was associated with the clinical tumor stage $(\mathrm{P}<0.05$; Table I). However, there was no association between RPN11 expression and age, tumor size, lymphatic vessel invasion, $\mathrm{T}$ classification or $\mathrm{N}$ classification.

Patients with high RPN11 expression exhibited significantly poorer overall survival, as determined by Kaplan-Meier analysis with $\log$-rank test $(\log$-rank=5.475; P=0.019; Fig. 1E). This was supported by the GSE1456 dataset, which was analyzed using PROGgene version 2.0 software (Indiana University, Indianapolis, IN, USA) $(18,19)$. High expression of RPN11 was associated with poorer survival compared with tissues with low RPN11 expression (Fig. 1F; $\mathrm{P}=0.0368$ ). In conclusion, mRNA and protein expression levels of RPN11 were upregulated in breast cancer tissues and this was associated with advanced cancer stage and poor patient prognosis.

RPN11 promotes cell growth and inhibits apoptosis in breast cancer cells. The effect of RPN11 inhibition on the MDA-MB-231 and T47D breast cancer cell lines was investigated. Knockdown of RPN11 reduced the proliferation of MDA-MB-231 and T47D cells compared with cells transfected with negative control siRNA, suggesting that RPN11 may serve a role in oncogenesis (Fig. 2A). In addition, the effect of RPN11 knockdown on apoptosis and the cell cycle was investigated. Flow cytometric analysis suggested that knockdown of RPN11 led to an enhanced degree of apoptosis (Fig. 2B) and suppression of the cell cycle, compared with cells transfected with negative control siRNA (Fig. 2C). Knockdown of RPN11 induced G0/G1 arrest (Fig. 2C). In addition, cyclin D1, a marker of the cell cycle, and cleaved PARP expression, a marker of apoptosis, were measured by western blot analysis. Knockdown of RPN11 enhanced cleaved-PARP and reduced cyclin D1 expression levels (Fig. 2D). This suggested that knockdown of RPN11 inhibited cell proliferation and induced apoptosis.

To further evaluate the role of RPN11 in breast cancer, RPN11 was exogenously overexpressed in MCF7 and Hs578T cells. Overexpression of RPN11 promoted cell growth $(\mathrm{P}<0.05$; Fig. 3A) and cell cycle progression (Fig. 3B); however, apoptosis was reduced compared with the vector control $(\mathrm{P}<0.05)$. 
A
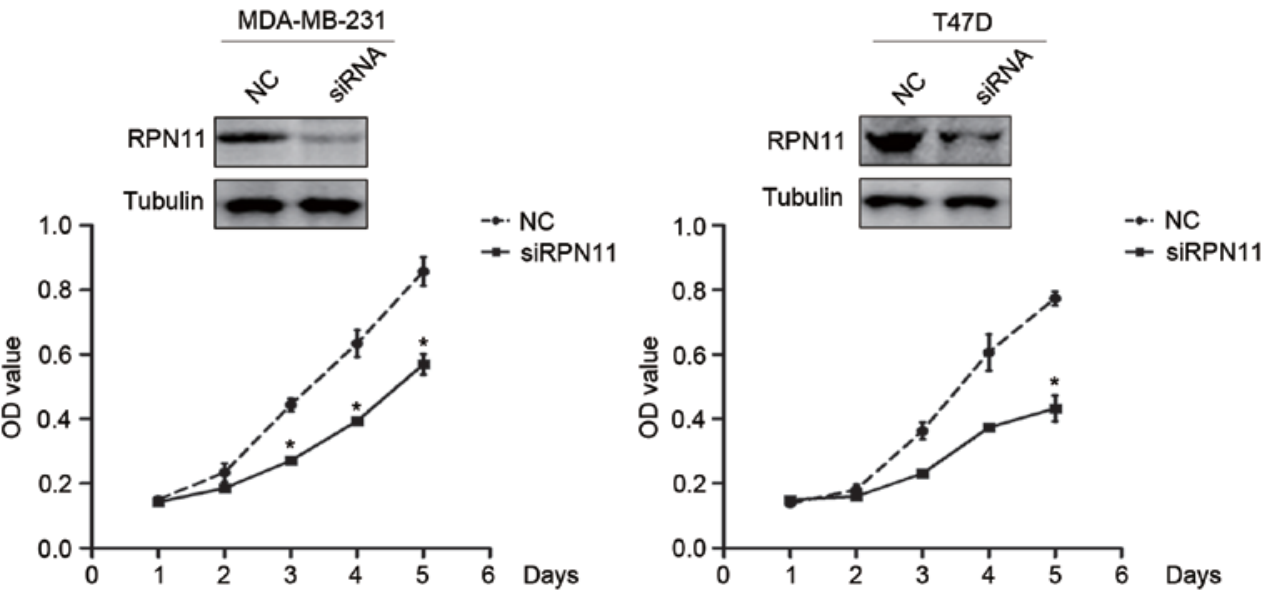

B
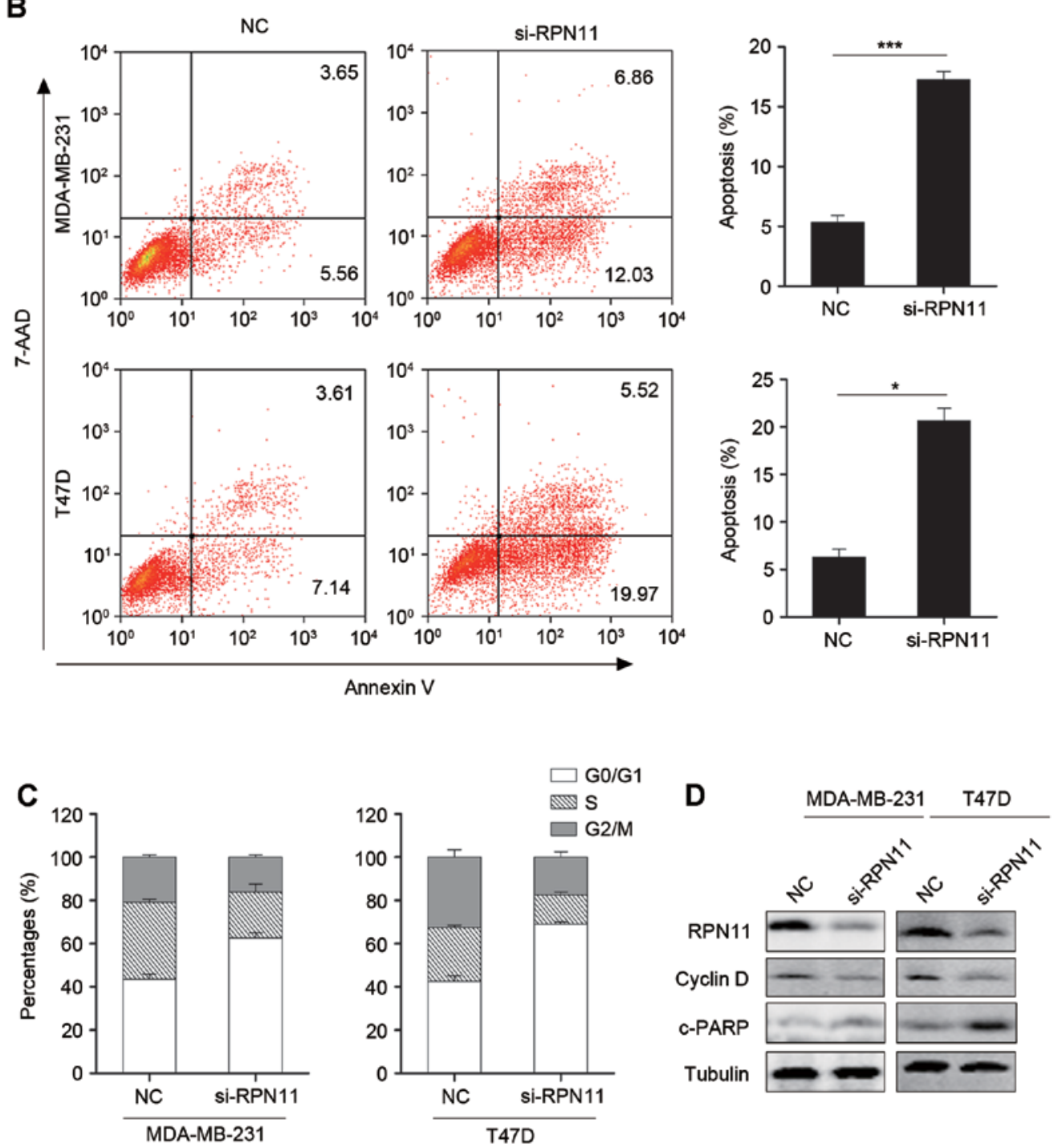

Figure 2. Knockdown of RPN11 induces cell cycle arrest and promotes apoptosis. (A) Cell proliferation assay was performed following transfection with si-RPN11 or NC siRNA in MDA-MB-231 and T47D cells. RPN11 knockdown by the siRNA pool was confirmed by western blot analysis. (B) Apoptosis was measured by Annexin V/7-AAD staining followed by flow cytometry. (C) Cell cycle analysis was performed following RPN11 knockdown in MDA-MB-231 and T47D cells. (D) RPN11, cyclin D, c-PARP and tubulin were measured by western blot analysis following RPN11 knockdown in MDA-MB-231 and T47D cells. Data are expressed as the mean \pm standard error of three independent experiments. ${ }^{*} \mathrm{P}<0.05 ;{ }^{* * * *} \mathrm{P}<0.001$. siRPN11, RPN11-targeting small interfering siRNA; c-PARP, cleaved-poly ADP ribose polymerase; NC, negative control; 7-AAD, 7-aminoactinomycin D.

This data suggested that RPN11 may have oncogenic potential and may be a therapeutic target.
RPN11 knockdown reduces breast cancer cell migration. Knockdown of RPN11 resulted in reduced cell migration in 

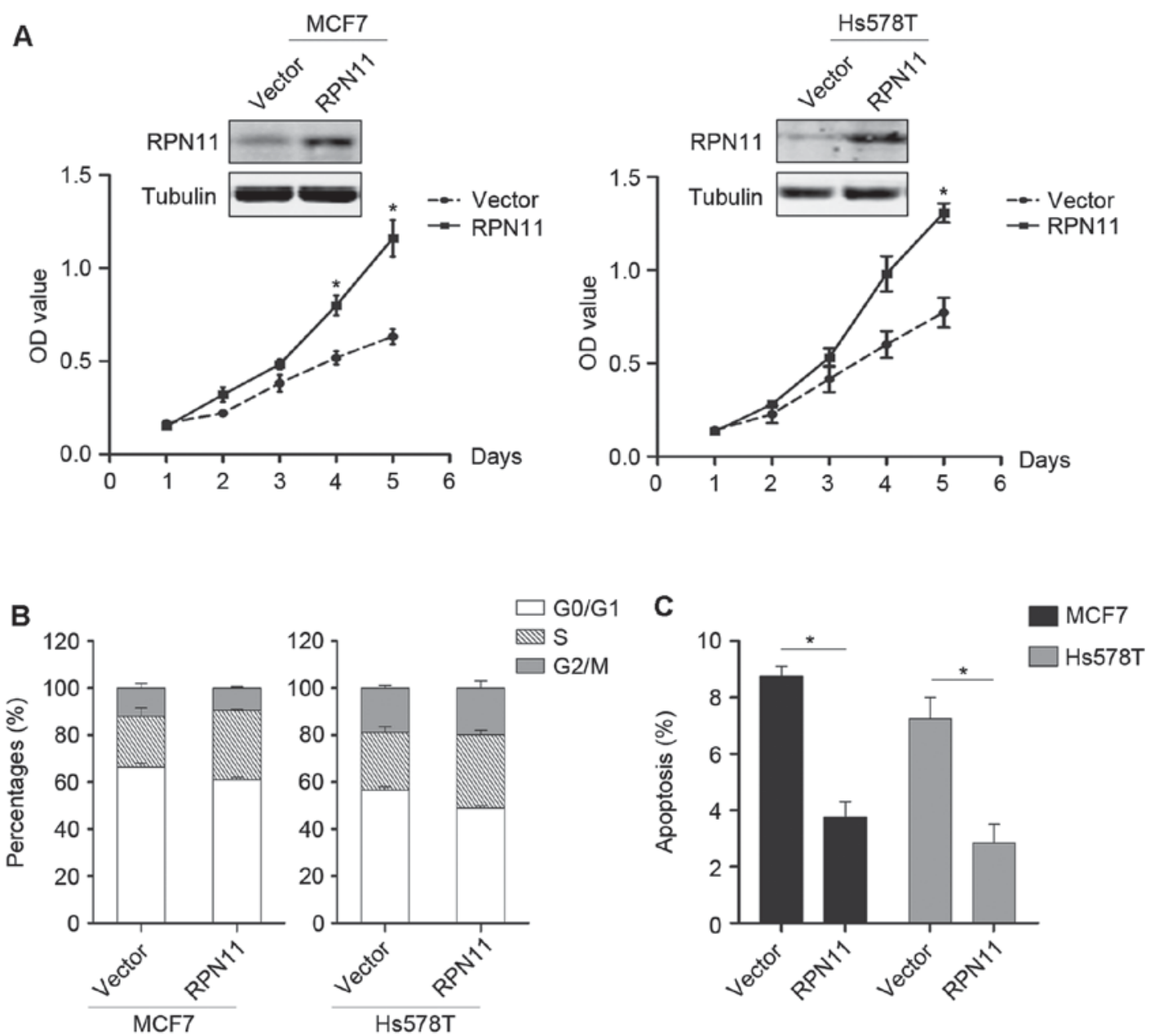

Figure 3. RPN11 overexpression promotes cell growth and inhibits apoptosis. (A) Cell proliferation assay was performed following transfection with the vector control or the RPN11 plasmid in MCF7 and Hs578T cells. RPN11 overexpression was confirmed by western blot analysis. (B) Analysis of the cell cycle and (C) apoptosis in MCF7 and Hs578T cells following RPN11 overexpression, as determined by flow cytometry. Data are expressed as the mean \pm standard error of three independent experiments. ${ }^{*} \mathrm{P}<0.05$. OD, optical density.

breast cancer cells compared with the negative control, as determined by a Transwell assay (Fig. 4A). EMT is a key step in tumor cell migration (20). Therefore, genes associated with EMT were measured by RT-qPCR following RPN11 knockdown. The level of mRNA encoding the epithelial marker E-cadherin was increased, whereas the levels of mRNA encoding the mesenchymal markers $\mathrm{N}$-cadherin and vimentin were reduced following knockdown of RPN11, compared with the negative control (Fig. 4B). The mRNA expression levels of EMT-associated transcriptional regulators, Snail and Slug, were reduced following RPN11 knockdown. In addition, western blot analysis corresponded with the RT-qPCR data (Fig. 4C). Collectively, the results suggested that overexpression of RPN11 in breast cancer may promote cancer cell migration.

\section{Discussion}

The mortality rate of breast cancer has declined due to improvements in earlier diagnosis and adjuvant therapies. Novel prognostic markers remain to be identified. carcinoembryonic antigen and carcinoma antigen 15-3 are widely recognized as tumor markers of breast cancer; however, the sensitivity and precision of the assays utilized for their detection requires further investigation (21-23). Therefore, it is necessary to identify novel markers for the diagnosis and treatment of patients with breast cancer.

The results of the present study indicated that RPN11 was significantly upregulated in breast cancer tissues compared with adjacent non-tumor tissues, and high expression of RPN11 was associated with the clinical tumor stage. Patients with high RPN11 expression levels had significantly poorer outcomes, which suggested that RPN11 may be a prognostic factor for patients with breast cancer. To validate these findings, the GSE1456 breast cancer cohort was analyzed. Results revealed that patients with high RPN11 expression had poor survival outcomes. In addition, in vitro experiments demonstrated that knockdown of RPN11 reduced proliferation and induced cell cycle arrest and apoptosis in breast cancer cells. By contrast, overexpression of RPN11 in breast cancer cells promoted cell growth and inhibited apoptosis.

Recently, RPN11 has been reported to function as an oncogene in a number of cancer types, including hepatocellular carcinoma and ovarian cancer (11). Wang et al (11) reported that RPN11 acts as a deubiquitinase enzyme and stabilizes the expression of E2F1 by removal of the polyubiquitin chain, 
A
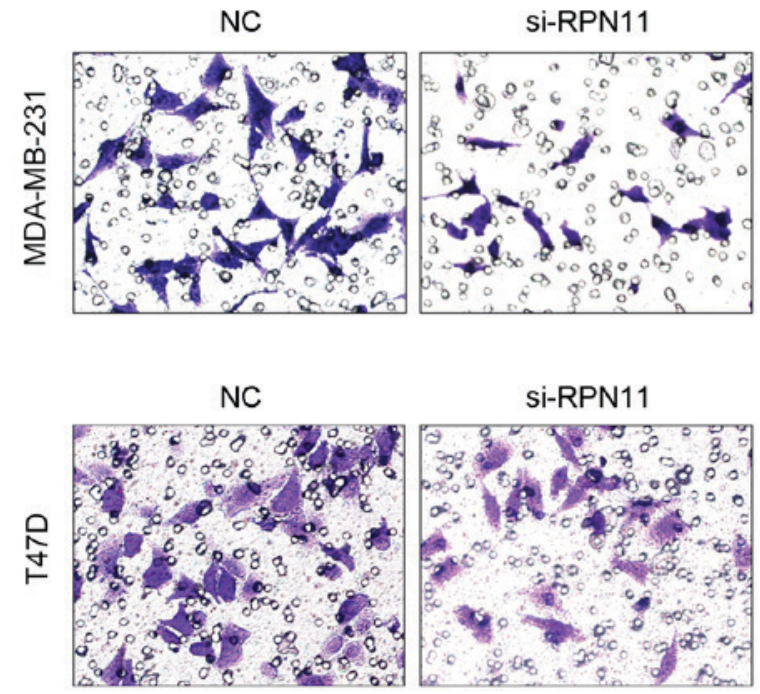
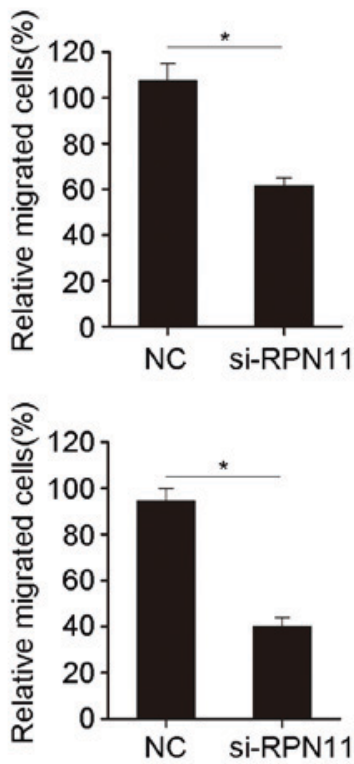

C
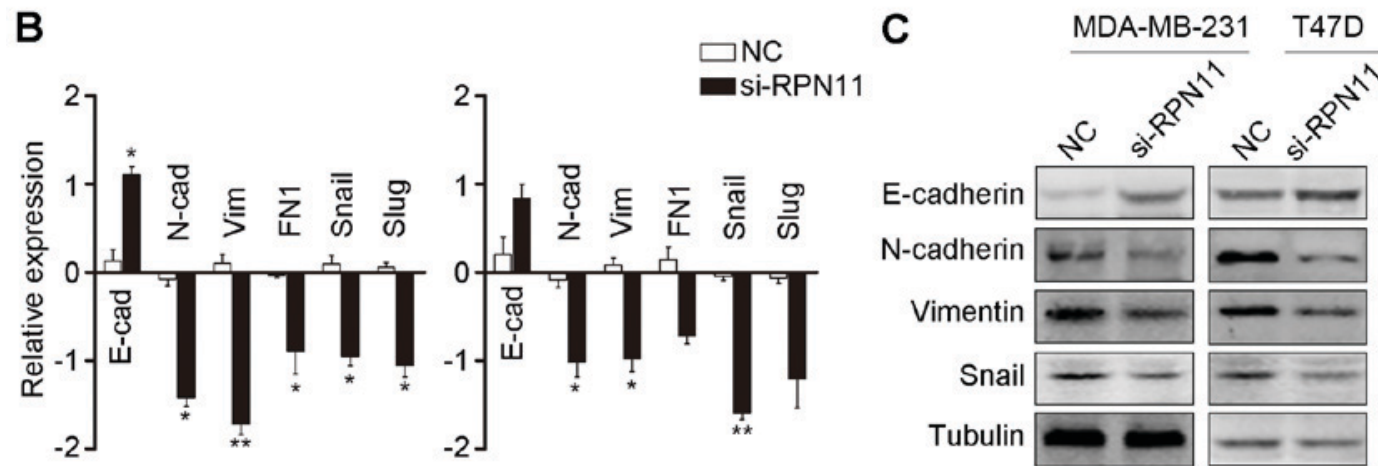

MDA-MB-231

Figure 4. Knockdown of RPN11 induces mesenchymal-epithelial transition in breast cancer cells. (A) Knockdown of RPN11 inhibited cell migration ability, as determined by the Transwell assay. (B) EMT markers and EMT-associated transcriptional factors, including E-cad, N-cad, vim, Fn1 Snail and Slug, were measured by reverse transcription-quantitative polymerase chain reaction following RPN11 knockdown in MDA-MB-231 and T47D cells. ${ }^{*}<0.05,{ }^{* *} \mathrm{P}<0.01$. (C) Protein expression levels of E-cad, N-cad, vim, Snail and tubulin were measured by western blot analysis following transfection with the NC or si-RPN11 in MDA-MB-231 and T47D cells. Data are expressed as the mean \pm standard error of three independent experiments. ${ }^{*} \mathrm{P}<0.05$. NC, negative control; siRPN11, RPN11-targeting small interfering siRNA; E-cad, E-cadherin; N-cad, N-cadherin; Vim, vimentin; FN1, fibronectin 1.

resulting in abnormal cell proliferation and tumorigenesis. In addition, it was demonstrated that expression of RPN11 was associated with the expression of survivin and forkhead box M1, both of which are considered to be oncogenes. Byrne et al (10) suggested that knockdown of RPN11 in HeLa cells enhanced cell cycle arrest and senescence by downregulation of cyclin B1-cell division cycle 25C and cyclin D1, and upregulation of p21, which was consistent with the findings of the present study. Furthermore, RPN11 promoted the double-strand DNA break response by processing of the polyubiquitin chain and recruiting p53 binding protein 1 to the site of DNA damage (24). RPN11 may specifically cleave lysine 63-linked polyubiquitin chains, which are critical for the regulation of substrates (25). Therefore, the downstream targets of RPN11 require further investigation.

The results of the present study suggested that RPN11 promotes cell migration by inducing EMT. Despite there being no clinical association between RPN11 expression and N classification, knockdown of RPN11 reduced cell migration by inducing mesenchymal-epithelial transition (MET). In EMT, cells lose their adhesion and epithelial features and switch to a mesenchymal phenotype, which is an indicator of cancer metastasis (26). In the present study, as the relative expression of RPN11 was higher in MCF-7 and Hs578T compared with MDA-MB-231 and T47D (data not shown), MCF-7 and H578T were used for the overexpression of RPN11 and MDA-MB-231/T47D cells were used for the knockdown. The Transwell assay suggested that knockdown of RPN11 partially abrogates the migration of breast cancer cells. To verify this, the expression levels of EMT-associated genes were measured, including E-cadherin, FN1, Snail and Slug. Results suggested that inhibition of RPN11 induced MET.

In conclusion, overexpression of RPN11 in breast cancer tissues was associated with an advanced clinical stage. Patients with tumors with high expression of RPN11 had worse prognosis. These findings suggest a role of RPN11 in breast cancer development, progression, cell proliferation and migration by inducing EMT. The data suggest that RPN11 may be a therapeutic target for breast cancer. 


\section{References}

1. Ferlay J, Soerjomataram I, Dikshit R, Eser S, Mathers C, Rebelo M, Parkin DM, Forman D and Bray F: Cancer incidence and mortality worldwide: Sources, methods and major patterns in GLOBOCAN 2012. Int J Cancer 136: E359-E386, 2015.

2. Siegel RL, Miller KD and Jemal A: Cancer statistics, 2015. CA Cancer J Clin 65: 5-29, 2015.

3. Saini KS, Loi S, de Azambuja E, Metzger-Filho O, Saini ML, Ignatiadis M, Dancey JE and Piccart-Gebhart MJ: Targeting the $\mathrm{PI} 3 \mathrm{~K} / \mathrm{AKT} / \mathrm{mTOR}$ and Raf/MEK/ERK pathways in the treatment of breast cancer. Cancer Treat Rev 39: 935-946, 2013.

4. Luga V, Zhang L, Viloria-Petit AM, Ogunjimi AA, Inanlou MR, Chiu E, Buchanan M,Hosein AN, Basik M and Wrana JL: Exosomes mediate stromal mobilization of autocrine Wnt-PCP signaling in breast cancer cell migration. Cell 151: 1542-1556, 2012.

5. Cai J, Guan H, Fang L, Yang Y, Zhu X, Yuan J, Wu J and Li M: MicroRNA-374a activates Wnt/ $\beta$-catenin signaling to promote breast cancer metastasis. J Clin Invest 123: 566-579, 2013.

6. Dvinge H, Git A, Gräf S, Salmon-Divon M, Curtis C, Sottoriva A, Zhao Y, Hirst M, Armisen J, Miska EA, et al: The shaping and functional consequences of the microRNA landscape in breast cancer. Nature 497: 378-382, 2013.

7. Kakarougkas A, Ismail A, Katsuki Y, Freire R, Shibata A and Jeggo PA: Co-operation of BRCA1 and POH1 relieves the barriers posed by 53BP1 and RAP80 to resection. Nucleic Acids Res 41: 10298-10311, 2013.

8. Stitzel ML, Durso R and Reese JC: The proteasome regulates the UV-induced activation of the AP-1-like transcription factor Gcn4. Genes Dev 15: 128-133, 2001.

9. Butler LR, Densham RM, Jia J, Garvin AJ, Stone HR, Shah V, Weekes D, Festy F, Beesley J and Morris JR: The proteasomal de-ubiquitinating enzyme $\mathrm{POH} 1$ promotes the double-strand DNA break response. EMBO J 31: 3918-3934, 2012.

10. Byrne A, McLaren RP, Mason P, Chai L, Dufault MR, Huang Y, Liang B, Gans JD, Zhang M, Carter K, et al: Knockdown of human deubiquitinase PSMD14 induces cell cycle arrest and senescence. Exp Cell Res 316: 258-271, 2010.

11. Wang B, Ma A, Zhang L, Jin WL, Qian Y, Xu G, Qiu B, Yang Z, Liu Y, Xia Q and Liu Y: POH1 deubiquitylates and stabilizes E2F1 to promote tumour formation. Nat Commun 6: 87804, 2015.

12. Gallery M, Blank JL, Lin Y, Gutierrez JA, Pulido JC, Rappoli D, Badola S, Rolfe M and Macbeth KJ: The JAMM motif of human deubiquitinase Poh1 is essential for cell viability. Mol Cancer Ther 6: 262-268, 2007.

13. Liu H, Buus R, Clague MJ and Urbé S: Regulation of ErbB2 receptor status by the proteasomal DUB POH1. PLoS One 4: e5544, 2009.
14. Nabhan JF and Ribeiro P: The $19 \mathrm{~S}$ proteasomal subunit POH1 contributes to the regulation of c-Jun ubiquitination, stability, and subcellular localization. J Biol Chem 281: 16099-16107, 2006.

15. Schwarz T, Sohn C, Kaiser B, Jensen ED and Mansky KC: The 19S proteasomal lid subunit POH1 enhances the transcriptional activation by Mitf in osteoclasts. J Cell Biochem 109: 967-974, 2010.

16. Livak KJ and Schmittgen TD: Analysis of relative gene expression data using real-time quantitative PCR and the 2(-Delta Delta C(T)) Method. Methods 25: 402-408, 2001.

17. Jiang J, Yu C, Chen M, Tian S and Sun C: Over-expression of TRIM37 promotes cell migration and metastasis in hepatocellular carcinoma by activating Wnt/ $\beta$-catenin signaling. Biochem Biophys Res Commun 464: 1120-1127, 2015.

18. Goswami CP and Nakshatri H: PROGgeneV2: Enhancements on the existing database. BMC Cancer 14: 970, 2014.

19. Goswami CP and Nakshatri H: PROGgene: Gene expression based survival analysis web application for multiple cancers. J Clin Bioinforma 3: 22, 2013.

20. Lamouille S, Xu J and Derynck R: Molecular mechanisms of epithelial-mesenchymal transition. Nat Rev Mol Cell Biol 15: 178-196, 2014.

21. Wu SG, He ZY, Zhou J, Sun JY, Li FY, Lin Q, Guo L and Lin HX: Serum levels of CEA and CA15-3 in different molecular subtypes and prognostic value in Chinese breast cancer. Breast 23: 88-93, 2014.

22. Lee JS, Park S, Park JM, Cho JH, Kim SI and Park BW: Elevated levels of preoperative CA 15-3 and CEA serum levels have independently poor prognostic significance in breast cancer. Annals of oncology 24: 1225-1231, 2013.

23. Van Poznak C, Somerfield MR, Bast RC, Cristofanilli M, Goetz MP, Gonzalez-Angulo AM, Hicks DG, Hill EG, Liu MC, Lucas W, et al: Use of biomarkers to guide decisions on systemic therapy for women with metastatic breast cancer: American Society of Clinical Oncology Clinical Practice Guideline. J Clin Oncol 2015: 2695-2704, 2015.

24. Morris JR: Attenuation of the ubiquitin conjugate DNA damage signal by the proteasomal DUB POH1. Cell Cycle 11: 4103-4104, 2012.

25. Cooper EM, Cutcliffe C, Kristiansen TZ, Pandey A, Pickart CM and Cohen RE: K63-specific deubiquitination by two JAMM/MPN+ complexes: BR ISC-associated Brcc36 and proteasomal Poh1. EMBO J 28: 621-631, 2009.

26. De Craene B and Berx G: Regulatory networks defining EMT during cancer initiation and progression. Nat Rev Cancer 13: 97-110, 2013. 